\title{
Discrete breathers in the crystal $\mathrm{CuAu}$
}

\author{
P.V. Zakharov ${ }^{1, \dagger}$, M.D. Starostenkov ${ }^{2}$, A.M. Eremin ${ }^{1}$, A.I. Cherednichenko ${ }^{1}$ \\ 'zakharovpvl@rambler.ru
}

${ }^{1}$ The Shukshin Altai State Humanities Pedagogical University, 53 Korolenko str., 659333, Biysk, Russia

${ }^{2}$ Altai State Technical University, 46 Lenin str., 656038, Barnaul, Russia

In the present work investigations of discrete breathers (DB) in CuAu crystal by means of molecular dynamics are performed. The phonon density of states of the considered crystal has been calculated in order to estimate the possible frequency intervals of DB existence. The function of initial conditions setting displacements and velocities of atoms in a closely packed atimic row at zero time moment for excitation of the DB with hard nonlinearty type have been proposed. It was demonstrated that a DB of hard nonlinearty type can be excited in a closely packed atomic raw of $\mathrm{Cu}$ atoms in [110] crystallographic direction. It was established that in case of amplitude values greater than $0,55 \AA$ increase of frequency upon amplitude growth is not observed. This fact can be explain by the energy transfer to heavy atoms sublattice in case of considerable initial atimic displacement of light atoms. Energy profile of the DB and its time evolution have been studied and main directions of DB energy radiation have been established. It was shown that a soft type $\mathrm{DB}$ can be excited in the system by lowering the comsidered mass of $\mathrm{Cu}$ atoms and application of strain. A soft type DB had frequencies withn the deformation induced gap of the phonon spectrum of the $\mathrm{CuAu}$ crystal. The breather is localized on one $\mathrm{Cu}$ atom and its established life time was about several tens of vibration periods. The energy localized on this breather was found to have values up to $0.9 \mathrm{eV}$ while a hard nonlinearity type $\mathrm{DB}$ involves 6 atoms of the closely packed raw and hconcentrates up to $2.1 \mathrm{eV}$ of energy.

Keywords: molecular dynamics, discrete breathers, quasi-breather, localization of energy, phonon spectrum.

\section{Дискретные бризеры в кристалле $\mathrm{CuAu}$}

\author{
Захаров П.В. ${ }^{1 \dagger}$, Старостенков М.Д. ${ }^{2}$, Ерёмин А.М. ${ }^{1}$, Чередниченко А.И. ${ }^{1}$ \\ †zakharovpvl@rambler.ru
}

${ }^{1}$ Алтайский государственный гуманитарно-педагогический университет, ул. Короленко 53, 659333, г. Бийск, Россия
${ }^{2}$ Алтайский государственный технический университет, пр. Ленина 46, 656038, г. Барнаул, Россия

Методом молекулярной динамики обнаружены дискретные бризеры (ДБ) в кристалле СuАu. Для анализа возможности существования ДБ был произведен расчет плотности фононных состояний рассматриваемого кристалла. Предложена функция формирования начальных условий для возбуждения дискретного бризера с жестким типом нелинейности в кристалле $\mathrm{CuAu}$. Показано, что он может быть получен в плотноупакованном ряду атомов Сu вдоль направления [110]. Проанализированы условия для возбуждения дискретного бризера, а также зависимость его частоты от амплитуды. Установлено, что при увеличении начальной амплитуды более $0,55 \AA$ из именение частоты практически не происходит. Объяснить это можно тем, что значительное отклонение атомов из положения равновесия приводит к возбуждению тяжелой подрешетки кристалла, через которую часть энергии рассеивается в фононную подсистему последней. Приведен энергетический профиль дискретного бризера с жестким типом нелинейности и его эволюция с течением времени. Установлены основные направления рассеивания энергии ДБ. Рассмотрены условия формирования дискретного бризера с мягким типом нелинейности, за счет деформации кристалла и занижения массы атомов меди. Установлено, что дискретный бризер с мягким типом нелинейности может существовать в щели фононного спектра кристалла $\mathrm{CuAu}$, образованной за счет деформации кристалла или занижения массы меди. Произведены расчеты плотности фононных состояний $\mathrm{CuAu}$ при деформациях и занижении массы атомов $\mathrm{Cu}$. Полученные результаты свидетельствуют, что ДБ с мягким типом нелинейности локализован в основном на одном атоме меди и существует несколько десятков периодов колебаний. Не смотря на это, он способен совокупно сосредотачивать энергию порядка 0,9 эВ, в то время как ДБ с жестким типом нелинейности в основном локализован на 6 атомах и их совокупная энергия составляет до 2,1 эВ.

Ключевые слова: молекулярная динамика, дискретный бризер, квази-бризер, локализация энергии, фононный спектр. 


\section{1. Введение}

Локализованные состояния атомов или групп атомов в кристаллах остаются слабо изучены в силу сложности проведения натурных экспериментов. При этом неуклонно растет число работ, посвященное данной тематике, в которых исследуются различные локализованные состояния атомов с помощью методов атомистического моделирования. Данная работа посвящена изучению локализованного объекта в кристалле - дискретного бризера (ДБ). Дискретный бризер - это нелинейный объект, который представляет собой локализованное в пространстве незатухающее колебание большой амплитуды в бездефектной нелинейной дискретной системе [1]. В кристаллах следует говорить о квази-бризерах, имеющих конечное время жизни и нестрогую периодичность колебаний во времени [2]. Авторы ряда работ высказывают мнения, что ДБ могут оказывать влияние на различные процессы в кристаллах. Так предполагается, что ДБ могут повышать каталитические свойства наночастиц с неупорядоченной структурой, приводить к радиационно-стимулированному отжигу (залечиванию) пор в металлах, вносить вклад в диффузию, транспортировать электрический заряд, приводить к отжигу дефектов, снижать энергетический барьер химических реакций в кристаллических твердых телах и т. д. [3-8].

ДБ имеют большое время жизни, так как их частота лежит вне фононного спектра кристалла из-за нелинейной зависимости частоты от амплитуды колебаний. Возможно два варианта такой зависимости. В первом случае, с увеличением амплитуды частота уменьшается, такие ДБ называют дискретными бризерами с мягким типом нелинейности или щелевыми дискретными бризерами. Такое название обусловлено тем, что их частота чаще всего попадает в запрещенную зону фононного спектра кристалла между акустической и оптической ветвями. ДБ с мягким типом нелинейности в основном локализуются на одном или двух атомах и не обладают мобильностью. Второй вариант зависимости, когда частота растет вместе с амплитудой, такие ДБ называют дискретными бризерами с жестким типом нелинейности. Частота ДБ с жестким типом нелинейности обычно располагается выше фононного спектра кристалла, такие ДБ могут перемещаться по кристаллу вдоль плотноупакованных рядов атомов. Щелевые ДБ могут возбуждаться в биатомных кристаллах [9-12], а также в графене и графане [13]. ДБ с жестким типом нелинейности обнаруживались в моноатомных системах и в чистых металлах [14].

\section{2. Описание модели и методика эксперимента}

Нами рассматривалась биатомная система $\mathrm{CuAu}, \mathrm{c}$ целью выявления различных типов дискретных бризеров в данном кристалле. В качестве метода был выбран метод молекулярной динамики, как хорошо зарекомендовавший себя в исследованиях таких процессов. Данный метод был реализован с помощью пакета молекулярно-динамического моделирования LAMMPS, где используются хорошо апробированные многочастичные межатомные потенциалы, построенные по методу погруженного атома (ЕАМ-потенциалы). При расчетах был применен потенциал авторов работы [15] для системы $\mathrm{Cu}-\mathrm{Au}$.

Для анализа возможности существования ДБ в кристалле $\mathrm{CuAu}$ был произведен расчет плотности фононных состояний рассматриваемого кристалла (см. рис. 1). Отсутствие щели в фононном спектре (ФС) CuAu говорит о том, что в нем маловероятно наличие дискретных бризеров с мягким типом нелинейности $[16,17]$.

Процесс поиска дискретных бризеров в кристаллах подразумевает подбор начальных условий - отклонений атомов из положения равновесия или задание начальных скоростей. В случае большой разности масс в биатомных кристаллах (более чем в 4 раза) достаточно вывести из положения равновесия в определенном направлении один или два атома, что приводит к формированию ДБ с мягким типом нелинейности. В рассматриваемом кристалле, такой подход не привел к формированию ДБ ни по одному из кристаллографических направлений в силу характерного фононного спектра, при нормальных условиях.

Для чистых металлов или сплавов с меньшим разбросом масс компонент условия возбуждения ДБ с жестким типом нелинейности более специфичны. Так авторами работы [18], был предложен анзац для возбуждения ДБ в чистых ГЦК и ОЦК металлах, подразумевающий задание профиля ДБ путем придания им смещений с помощью особых функций. Смещения атомов осуществлялись таким образом, что соседние атомы осуществляли колебания в противофазе [18].

В данной работе для создания начального профиля ДБ использовалась функция Гаусса (1), адаптированная для условий кристалла

$$
f(x)=A_{0} e^{-\frac{(x-b)^{2}}{2 C^{2}}} .
$$

Из этого уравнения был исключен параметр b, обеспечивающий смещение максимума функции вдоль ряда атомов, т. к. рассматривался идеальный кристалл без топологических дефектов, таким образом, имеем функцию вида:

$$
f(x)=A_{0} e^{-\frac{x^{2}}{2 C^{2}}},
$$

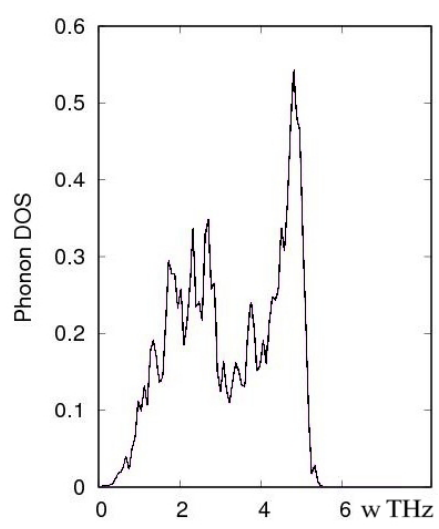

Рис. 1. Плотность фононных состояний кристалла $\mathrm{CuAu}$. Fig. 1. The density of phonon states CuAu crystal. 
где $A_{0}$ задает начальную амплитуду центральных атомов дискретного бризера, $x$ - относительная координата пары атомов в ряду, параметр $C$ степень пространственной локализации дискретного бризера. Варьируя значение $A_{0}$ и $C$ подбираем профиль дискретного бризера, тем самым задавая начальные отклонения из положения равновесия атомам, входящим в ДБ (см. рис. 2).

\section{3. Результаты и обсуждение}

Рассматривая кристалл $\mathrm{CuAu}$ на возможность формирования ДБ с жестким типом нелинейности были проверены плотноупакованные направления для подрешеток $\mathrm{Cu}$ и $\mathrm{Au}$. Дискретный бризер удалось получить вдоль направления [110] для атомов меди. Существование ДБ на атомах $\mathrm{Au}$ не возможно, так как их отклонение из положения равновесия приводит к быстрому возбуждению соседних атомов более легкой подрешетки $\mathrm{Cu}$, что в свою очередь влечет рассеивание энергии по кристаллу.

Наиболее продолжительные колебания удалось получить для параметров уравнения (2) $A_{0}=0,48 \AA$, $C=0,75$. Время жизни ДБ в этом случае превысило 50 пс. Сформированный дискретный бризер был локализован на 6 - 8 атомах меди, колеблющихся в противофазе.

Наиболее важной характеристикой ДБ является зависимость его частоты от амплитуды колебаний атомов. Варьируя $A_{0}$ в уравнении (2) и снимая результаты с модели, была получена соответствующая кривая, представленная на рис. 3. Фиксация частоты колебаний осуществлялась через 20-30 колебаний с начала эксперимента. Как видно из рис. 3 , при увеличении начальной амплитуды более 0,55 А̊ изменение частоты практически не происходит. Объяснить это можно тем, что значительное отклонение атомов из положения равновесия приводит к возбуждению тяжелой подрешетки кристалла, через которую часть энергии рассеивается в фононную подсистему последней. Дальнейшее увеличение начальной амплитуды приводило к полному рассеиванию энергии по кристаллу без образования дискретного бризера.

Не менее важной величина является энергии, которую ДБ способен локализовать на себе продолжительное

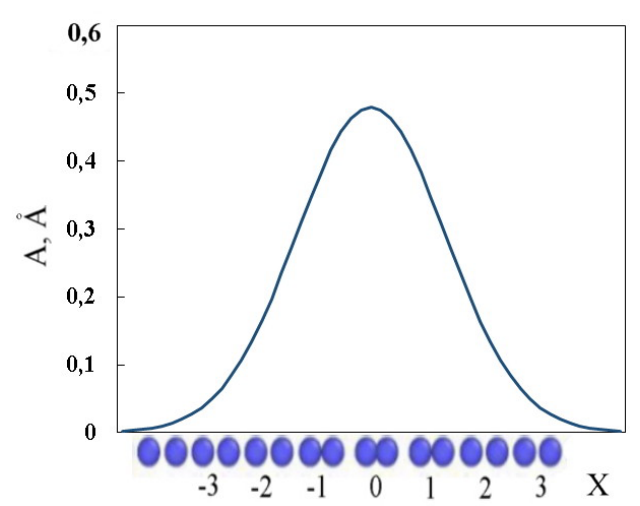

Рис. 2. Начальный профиль дискретного бризера, задаваемый функцией (2) для ряда меди вдоль направления [110].

Fig. 2. Basic profile discrete breather given by the function (2) for a number of copper along the [110]. время. На рис.4. приведена зависимость энергии дискретного бризера с жестким типом нелинейности от амплитуды колебаний центральных атомов ДБ, которую он способен сохранять не менее 5 пс.

Степень пространственной локализации ДБ в течение его существования можно оценить по энергетическому профилю, представленному на рис. 5. Дискретный бризер локализован на 6-8 атомах $\mathrm{Cu}$ и может совокупно сосредотачивать до 2,1 эВ.

В ряде работ [19-22] было показано, что дискретный бризер с мягким типом нелинейности возможно получить в кристаллах путем их деформации или занижения массы компонента сплава, что приводило к формированию щели в фононном спектре кристалла, тем самым обеспечивая необходимое условия для существования ДБ.

Нами производилась деформация кристалла $\mathrm{CuAu}$ с учетом принципа Пуассона, т.е. объем модельной ячейки сохранялся. Характерным показателем для кристаллов с тетрагональной структурой является отношение параметров решетки $c / a$, для $\mathrm{CuAu}$ при нормальных условиях это соотношение равно 0,92. Деформируя кристалл вдоль кристаллографического направления [001], соответствующего параметру решетки $c$, учитывая изме-

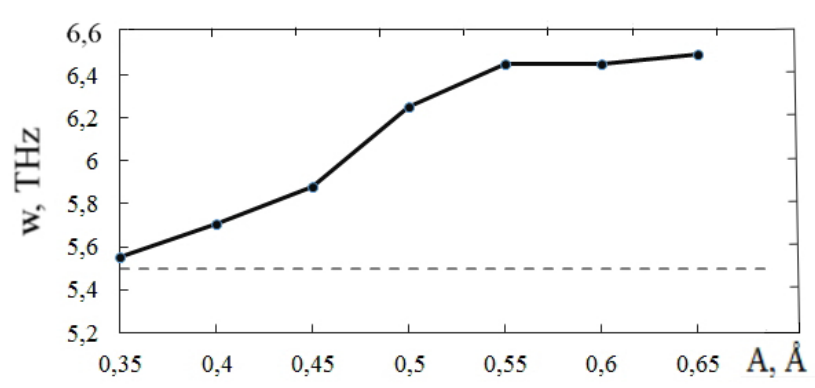

Рис. 3. Зависимость частоты $\omega$ колебаний атомов, входящих в дискретный бризер, от амплитуды $A$ центральных атомов ДБ; пунктирной линией показана верхняя граница фононного спектра кристалла $\mathrm{CuAu}$.

Fig. 3. The frequency $\omega$ vibrations of the atoms belonging to the discrete breathers, the amplitude $A$ of the central atoms DB; the dashed line shows the upper limit of the phonon spectrum of the crystal CuAu.

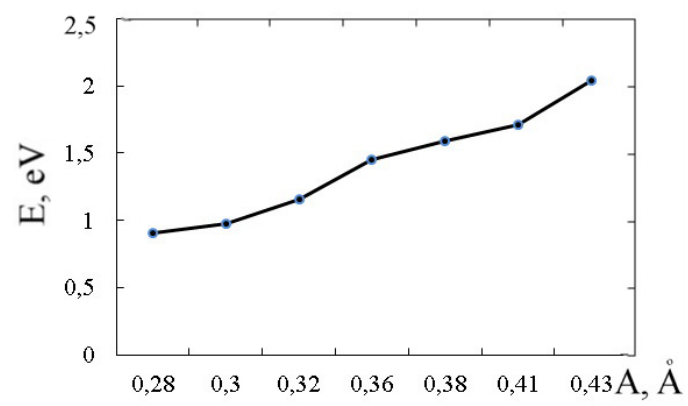

Рис. 4. Зависимость энергии дискретного бризера с жестким типом нелинейности от амплитуды колебаний центральных атомов.

Fig. 4. The dependence of the energy of the discrete breather with a hard type of the nonlinearity of the amplitude of oscillation of the central atoms. 


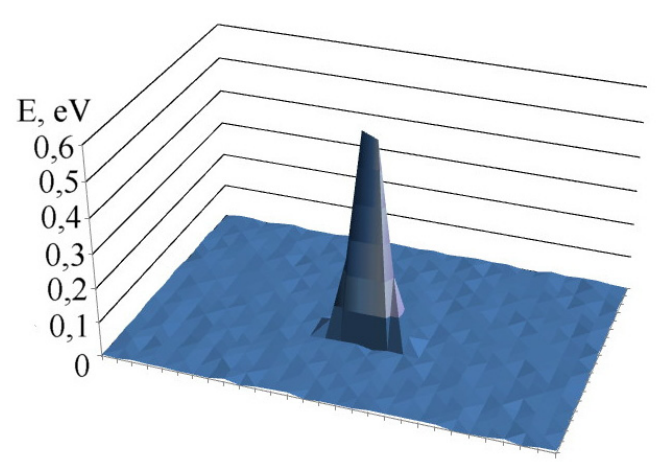

a

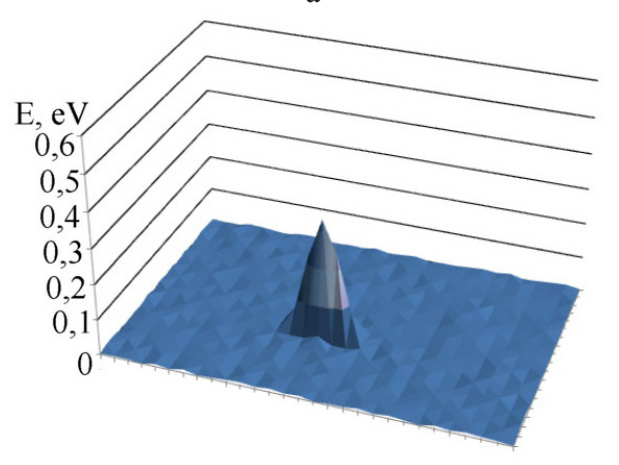

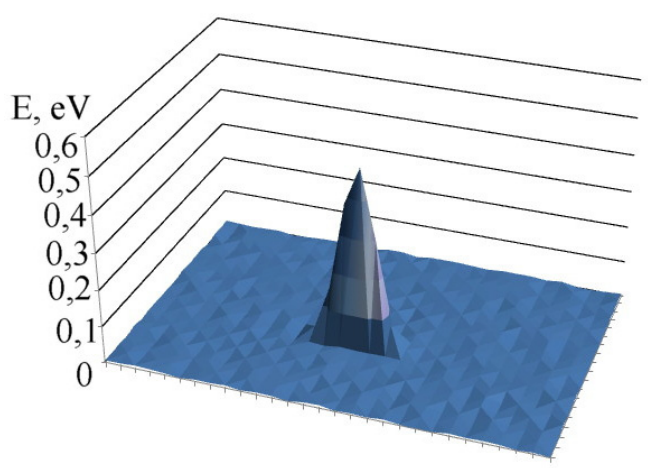

$\mathrm{b}$

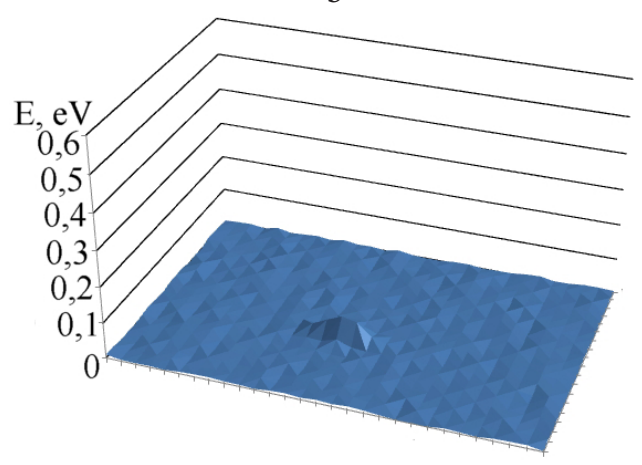

$\mathrm{d}$

Рис. 5. Эволюция энергетического профиля дискретного бризера с жестким типом нелинейности: а) в момент времени 5 пс от начала эксперимента, полная энергия ДБ 1,71 эВ, b) в момент времени 30 пс от начала эксперимента, полная энергия ДБ 1,45 эВ, c) в момент времени 45 пс от начала эксперимента, полная энергия ДБ 0.98 эВ, d) в момент времени 50 пс от начала эксперимента, полная энергия ДБ 0,25 эВ.

Fig. 5. Evolution of the energy profile of the discrete breather with a hard type of non-linearity: a) Under the instant 5 pc from the beginning of the experiment, the total energy of $1.71 \mathrm{eV} \mathrm{DB}, \mathrm{b}$ ) As at time of $30 \mathrm{ps}$ from the start of the experiment, the total energy of $1.45 \mathrm{eV} \mathrm{DB}, \mathrm{c})$ at the time of 45 ps from the start of the experiment, the total energy of $0.98 \mathrm{eV} \mathrm{DB}, \mathrm{d}$ ) into the time of 50 ps time from the beginning of the experiment, the total energy of $0.25 \mathrm{eV}$ DB.

нения параметра решетки $а$ для сохранения объема рассматриваемой модели, получали плотности фононных состояний кристалла.

Достаточно широкая щель в фононном спектре кристалла была получена при $c / a=0,8$, что составляло 9,5\% деформации сжатия вдоль направления [001] (см. рис. 6).

Дальнейшее увеличение деформации сжатия приводило к уширению щели, но в тоже время отрицательно влияло на стабильность модели.

При деформации растяжения кристалла $\mathrm{CuAu}$, т.е. при увеличении соотношения $c / a$, щели в фононном

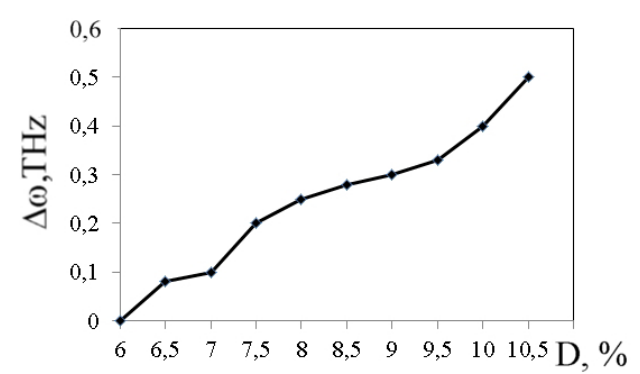

Рис. 6.Зависимость ширины щели фононного спектра кристалла $\mathrm{CuAu} \Delta \omega$ от величины деформации $D$ вдоль направлении [001].

Fig. 6. Dependence of the gap width of the phonon spectrum of the crystal CuAu $\Delta \omega$ the magnitude of deformation $D$ along the [001] direction. спектре кристалла не возникало.

Для поиска дискретного бризера с мягким типом нелинейности отклонялся из положения равновесия один из атомов $\mathrm{Cu}$ вдоль различных кристаллографических направлений. Как и ожидалось, в этом случае удалось получить дискретный бризер вдоль направления [100]. Частота полученного ДБ с мягким типом нелинейности лежит в щели фононного спектра и соответствует 3,5 ТГц. Время жизни полученных локализованных колебаний составляет порядка 2 пс или более 15 периодов колебаний. Такой тип ДБ локализован в основном на одном атоме $\mathrm{Cu}$. В силу узкой щели в фононном спектре получить зависимость частоты от амплитуды в широком диапазоне не представляется возможным.

Далее были проведены эксперименты с заниженной массой меди без деформации. Аналогичной щели в ФС, как на рис. 7, удалось достичь при уменьшении массы $\mathrm{Cu}$ до 36 a.e.m. (нормальная 63,546 a.e.m.) или это соответствовало отношению массы золота к меди равное 5,5 (см. рис. 8). Продолжительность локализованных колебаний в этом случае была аналогичной, порядка 15 периодов колебаний, что, видимо, обусловлено геометрией кристалла.

Деформация сжатия совместно с уменьшением массы атома меди до 20 a.e.m. привели к формированию более широкой щели в ФС при условии устойчивости кристаллической решетки, что позволило увеличить 
продолжительность жизни ДБ с мягким типом нелинейности до 100 периодов колебаний.

Для этого случая была рассчитана энергия, локализованная на ДБ с мягким типом нелинейности. Степень пространственной локализации и энергетический профиль ДБ с мягким типом нелинейности представлен на рис. 9.

Полученные результаты свидетельствуют, что ДБ с мягким типом нелинейности локализован в основном на одном атоме меди. Не смотря на это, он способен совокупно сосредотачивать энергию порядка 0,9 эВ, в то время как ДБ с жестким типом нелинейности в основном локализован на 6 атомах и их совокупная энергия составляет до 2,1 эВ.

\section{4. Заключение}

Таким образом, методом молекулярной динамики продемонстрирована возможность возбуждения дискретного бризера с жестким типом нелинейности в кристалле $\mathrm{CuAu}$ на подрешетке меди. Получена его зависимость частоты от амплитуды, подобраны оптимальные пара-

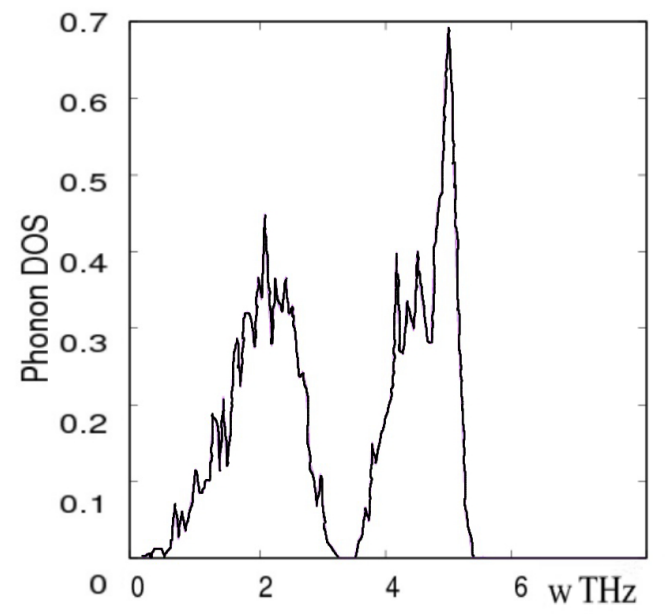

Рис. 7. Плотность фононных состояний кристалла $\mathrm{CuAu}$, при $c / a=0,8$ (деформация сжатия составила 9,5\%).

Fig. 7. The density of phonon states of the crystal $\mathrm{CuAu}$, with $c / a=0.8$ (compression deformation was $9.5 \%$ ). метры функции, описывающие первоначальный профиль дискретного бризера. Рассмотрена пространственная локализация и приведен энергетический профиль бризера, позволяющий оценить величину энергии ДБ. Установлено, что при деформации сжатия возможно формирование щели в фононном спектре кристалла $\mathrm{CuAu}$ и существование в ней дискретного бризера с мягким типом нелинейности на атомах $\mathrm{Cu}$. Получена зависимость ширины щели от приложенной деформации вдоль кристаллографического направления [001]. Показано, что искусственное занижение массы атомов меди, также приводит к щели в фононном спектре и созданию условий для существования ДБ с мягким типом нелинейности, локализованного вдоль направления [100]. При деформации или занижении массы, время жизни ДБ составляет величину порядка нескольких десятков периодов колебаний, для увеличения продолжительности колебаний к кристаллу прикладывалась деформация и занижалась масса атомов меди совместно. Для этого случая получена энергии ДБ, приведена эволюция энергетического профиля ДБ и его пространственная локализация.

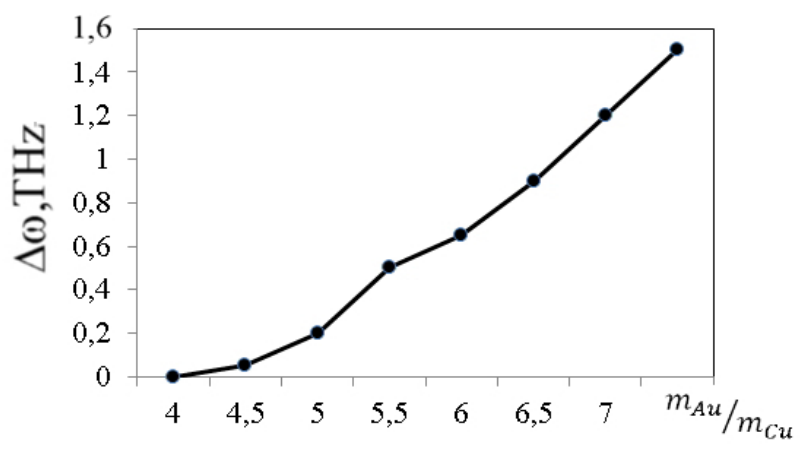

Рис. 8. Зависимость ширины щели фононного спектра кристалла $\mathrm{CuAu} \Delta \omega$ от величины отношения масс компонент сплава $\mathrm{Au}$ к $\mathrm{Cu}$.

Fig. 8. Dependence of the gap width of the phonon spectrum of the crystal $\mathrm{CuAu} \Delta \omega$ on the ratio of the masses Au alloy component to the $\mathrm{Cu}$.

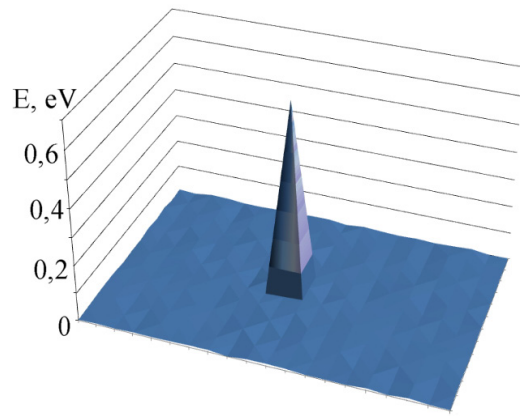

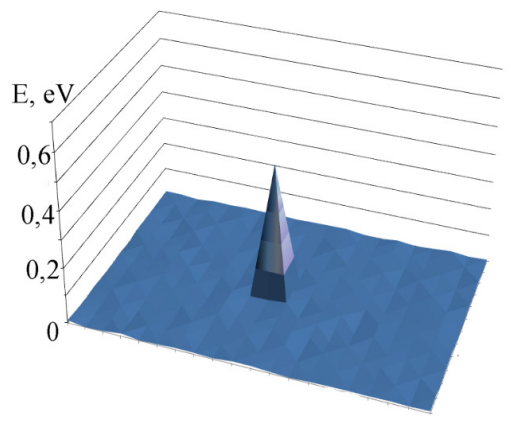

b

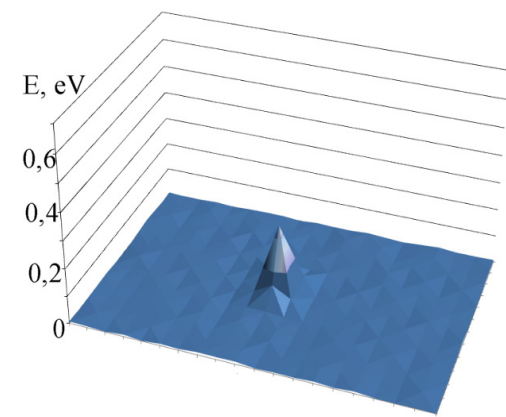

Рис. 9. Эволюция энергетического профиля дискретного бризера с мягким типом нелинейности: а) в момент времени 1 пс от начала эксперимента, полная энергия ДБ 0,72 эВ, b) в момент времени 2,5 пс от начала эксперимента, полная энергия ДБ 0,53 эВ, c) в момент времени 7 пс от начала эксперимента, полная энергия ДБ 0,28 эВ.

Fig. 9. The evolution of the energy profile of the discrete breather with a mild type of the nonlinearity: a) under point in time of 1 ps from the beginning of the experiment, the total energy of $0.72 \mathrm{eV} \mathrm{DB}, \mathrm{b}$ ) at time of $2.5 \mathrm{ps}$ from the beginning of the experiment, the total energy of $0.53 \mathrm{eV} \mathrm{DB}, \mathrm{c}$ ) at time $7 \mathrm{ps}$ from the beginning of the experiment, the total energy of $0.28 \mathrm{eV} \mathrm{DB}$. 
Благодарность/Acknowledgements. ЗПВ благодарит за финансовую поддержку РНФ проект № 16-12-10175.; ЕАМ благодарит за финансовую поддержку РФФИ проeкm № 16-42-220002 p_a.

\section{Литература/References}

1. A. J. Sievers, S. Takeno. Phys. Rev. Lett. 61, 970 (1988).

2. G. M. Chechin, G.S. Dzhelauhova, E.A. Mehonoshina. Phys. Rev. E. 74, 036608 (2006).

3. V.I. Dubinko, F. Piazza. Letters on Materials. 4 (4), $273-278$ (2014).

4. A. A. Stepanov, S. V. Dmitriev, A. S. Semenov, V. I. Batons, D. A. Terentyev. Technical Physics Letters. 40 (15), 58 - 65 (2014). (in Russian) [А.А. Кистанов, С.В. Дмитриев, А. С. Семенов, В. И. Дубинко, Д. А. Терентьев. Письма в ЖТФ. 40 (15), 58 - 65 (2014).]

5. M. G. Velarde. J. Comput. Appl. Math. 233, 1432 (2010)

6. J. F. R. Archilla, S. M. M. Coelho, F. D. Auret, V. I. Dubinko, V. Hizhnyakov. Physica D. 297, 56-61 (2015).

7. V.I. Dubinko, A. V. Dubinko. Nucl. Instrum. Methods B. 303, 133 - 135 (2013).

8. A.A. Kistanov, S.V. Dmitriev, A.P. Chetverikov, M. G. Velarde. Eur. Phys. J. B. 87 (9), 211 (2014).

9. N.N. Medvedev, M.D. Starostenkov, M.E. Manley. J. Appl. Phys. 114, 213506 (2013).

10. P. V. Zakharov, M.D. Starostenkov, S. V. Dmitriev. Key Engineering Materials. 685, 65 - 69 (2016).

11. M.D. Starostenkov, A.I. Potekaev, S.V. Dmitriev, P.V. Zakharov, A.M. Eremin, V.V. Kulagina. Russian Physics Journal. 58 (9), 1353-1357 (2016)
[М.Д. Старостенков, А.И. Потекаев, С. В. Дмитриев, П.В. Захаров, А.М. Ерёмин, В.В. Кулагина. Изв. вузов. Физика. 58 (9), 136 - 140 (2015).]

12. P.V. Zakharov, M.D. Starostenkov, N.N. Medvedev, A.M. Eremin et al. Fundamental problems of modern materials. 11 (4), 533-536 (2014). (in Russian) [П.В. Захаров, М.Д. Старостенков, Н.Н. Медведев, А. М. Ерёмин и др. ФПСМ. 11 (4), 533 - 536 (2014).]

13. B. Liu, J. A. Baimova, S. V. Dmitriev, X. Wang, H. Zhu, K. Zhou. J. Phys. D. 46, 305302 (2013).

14. R.T. Murzaev, A.A. Kistanov, V.I. Dubinko, D. A. Terentyev, S. V. Dmitriev. Comp. Mater. Sci. 98, $88-92$ (2015).

15. L. Ward, A. Agrawal, K.M. Flores, W. Windl. arXiv:1209.0619

16. Yu.S Kivshar, G. P. Agrawal. Optical Solitons: From Fibers to Photonic Crystals Academic. (2003).

17. S. V. Dmitriev. Letters of materials. 1, $78-83$ (2011). (in Russian) [С. В. Дмитриев. Письма о материалах. 1, $78-83$ (2011).]

18. A.A. Kistanov, R. T. Murzaev, S. V. Dmitriev, V.I. Batons, V.V. Khizhnyakov. JETP Lett. 99 (6), 403 (2014). (in Russian)

19. E. A. Korznikova, J. A. Baimova, S. V. Dmitriev. Europhys. Lett. 102, 60004 (2013)

20. E. A. Korznikova et al. JETP Lett. 96, 222 (2012)

21. S. V. Dmitriev, E.A. Korznikova, Y.A. Baimova, M.G. Velarde. Discrete breathers in crystals PhysicsUspekhi 59 (5), 446-461 (2016).

22. S. V. Dmitriev. Journal of Micromechanics and Molecular Physics 01 (02) 1630001 (July 2016) 